\title{
Los pactos territoriales en Italia. Un análisis de la experiencia reciente y sus implicancias en términos de governance
}

\begin{abstract}
The article analyses the importance of territorial pacts in Italy as a new trend in public policies to promote collective and negotiate processes of local development. This kind of instrument is considered from different stand-points: a) in the light of new tendencies and governance in the European scene, and b) related with the main results in terms of performance and efficiency in the Italian context. Finally, the study of these elements aims at considering the true relevance of pacts in the overall improvement of territory and the development of more pluralist practices at local levels.
\end{abstract}

Keywords: territorial pacts, public policy, governance, local development.

\section{Resumen}

El artículo aborda la importancia que alcanzan los pactos territoriales en Italia como nueva modalidad de política pública orientada a promover procesos colectivos y negociados de desarrollo local. Este tipo de instrumento se analiza desde distintas perspectivas: a) en el marco de las nuevas orientaciones de política y governance del escenario europeo, y b) en relación a los principales resultados obtenidos en términos de desempeño y eficacia para el contexto italiano. Con el estudio de estos elementos se pretende, finalmente, considerar la verdadera significación que alcanzan los pactos en el mejoramiento integral del territorio y en el desarrollo de prácticas más pluralistas y concertadas a nivel local.

Palabras claves: pactos territoriales, políticas públicas, governance, desarrollo local. 


\section{Introducción}

$\mathrm{E}$ n los últimos años ha habido en la política italiana un fuerte interés por estimular procesos concertados y estratégicos de desarrollo local. Uno de los instrumentos más novedosos para la concreción de estos objetivos son los pactos territoriales, como partes de un sistema de intervención integral que apunta a crear condiciones favorables para el crecimiento económico y la ocupación en una determinada área geográfica. Los pactos territoriales se dirigen fundamentalmente a promover la cooperación entre sujetos públicos y privados de un territorio determinado de modo de estimular el diseño y la realización de proyectos de mejoramiento del contexto local en el sector industrial, servicios y a nivel de infraestructura.

El pacto territorial es un instrumento de concertación de todas las fuerzas económicas y sociales presentes en el ámbito local con el fin de dirigirlas a la realización de objetivos comunes de desarrollo. Con esta herramienta, basada en la programación negociada, los distintos actores individualizan una serie de objetivos, seleccionando los proyectos de intervención compatibles con tales propósitos y concordando un conjunto de medidas para facilitar y sostener la ejecución de los mismos.

En este escenario, el trabajo busca en primer lugar analizar conceptualmente la importancia que asumen en el contexto europeo las nuevas orientaciones de política y governance en el plano territorial. Seguidamente, se consideran las particularidades que asume el marco institucional italiano para el despliegue de este tipo de instrumentos descentralizados y se analizan los principales elementos que caracterizan la actuación y eficacia de los pactos territoriales en términos de cobertura demográfica y territorial, eficiencia financiera, tipo de intervención en el territorio, actividades concluidas, grado de ocupación y niveles de governance desarrollados. Finalmente, se examina la significación que alcanza el fenómeno de

Investigador-docente del Área "Estado, Gobierno y Administración Pública” del Instituto del Conurbano (ICO) de la Universidad Nacional de General Sarmiento (UNGS), Provincia de Buenos Aires (Argentina). E-mail: rcarmona@ungs.edu.ar

* Recibido el 1 de marzo de 2005, aprobado el 20 de enero de 2006. los pactos en términos de superar las desventajas localizadas territorialmente y promover procesos genuinos de desarrollo local.

\section{Nuevas perspectivas de política y governance a nivel territorial}

Gran parte de la literatura actual sobre la cuestión regional y local en Europa viene planteando la importancia que asumen, en un marco de globalización, las nuevas orientaciones de política para el fortalecimiento del desarrollo y la innovación en el plano territorial. En este escenario, asumen una fuerte significación las formas en las que se involucran los diversos sectores e instituciones de la sociedad civil en ese tipo de políticas, y la importancia de considerar el pasaje desde una autoridad de gobierno claramente distinguible (government) a un acercamiento basado en formas más legítimas y concertadas de gobernar (governance), en las cuales participa una pluralidad de actores públicos, privados y de relevancia colectiva.

El nuevo enfoque de intervención regional que se desarrolla en los países europeos desde la última década se caracteriza por políticas orientadas a desarrollar interacciones entre agentes en espacios territoriales acotados, a los fines de incentivar los procesos de especialización y complementación recíproca entre los actores. En ese contexto, se considera a la creación de redes como el factor central para el éxito de las políticas, las que surgen como resultado de la interacción y cooperación entre los distintos niveles de gobierno (nacional, regional y local) y los agentes e instituciones públicas y privadas que operan en el sector socio-productivo (empresas, cámaras empresariales, centros tecnológicos, universidades, ONGs, etc.). Este énfasis en el desarrollo y fortalecimiento de las capacidades de los actores para participar de las políticas se pone en evidencia principalmente en los programas dirigidos al mejoramiento de los recursos humanos, el aprendizaje colectivo y el impulso de los procesos de innovación institucional y productiva, pensados como fundamentales para un mayor nivel de eficiencia y competitividad (Bennett y Krebs, 1994; Courlet y Soulage, 1995, Boscherini y Poma, 2000).

Este enfoque se distingue por ser un diseño orientado de abajo hacia arriba y tener una profun- 
da interrelación entre el plano estatal y privado. En tal sentido, se pasa a considerar no sólo la relevancia de un enfoque de política orientado desde arriba (top-down), sino también las acciones impulsadas por las instituciones y agentes locales (botton-up). Esto implica el tránsito de una política centralizada en un Estado que concentra el diseño y la implementación de la intervención, hacia un enfoque más descentralizado en su ejecución que asigna un rol preponderante a los actores y autoridades regionales y locales en un contexto de gran interacción con las instituciones centrales. Otro de los aspectos tiene que ver con que los procesos de reconversión de las instituciones y agentes no son percibidos como automáticos, sino que necesitan de nuevas formas de intervención pública y governance, en las que se replantea el rol del Estado, del sector privado y los distintos actores de la sociedad civil (Bianchi, 1997; Amin, 1999).

Con el término governance se pretende definir un nuevo estilo decisional y de gobierno, diferente a los modelos tradicionales más centralizados y jerárquicos, caracterizado por la interacción y la cooperación de los diferentes niveles gubernamentales, organizaciones sociales y actores privados en el desarrollo de las decisiones. Esto supone considerar la importancia que alcanzan nuevas formas de institucionalidad (tanto formal como informal) y generación de consenso en la determinación de una política pública particular en tanto actores, procedimientos y medios legítimos de acción colectiva. Como plantean Brugué, Goma y Subirats (2002: 409), "la nueva governance, por tanto, responde a la complejidad del momento asumiendo y provocando una doble complejidad: operar desde la interacción vertical (multinivel) y desde la incorporación de un número creciente de actores (red)".

De esta forma, la governance se relaciona a estructuras y procesos a través de los cuales una variedad de actores (políticos, sociales y económicos) desarrolla diversos niveles de articulación y toma de decisiones sobre asuntos de interés público. Desde este punto de vista, el enfoque se distingue por un mayor grado de interacción y cooperación entre el Estado y los actores no estatales, en un contexto de redes decisionales mixtas (Mayntz, 2001; Pierre, 2000).
Las modalidades de acción sintetizadas por esos modelos de governance estarían indicando, por lo tanto, una transformación del ejercicio del gobierno hacia prácticas más pluralistas que redefinen la calidad y el tipo de intervención pública en los territorios, favorecen la construcción de nuevas relaciones y conforman un proceso abierto a un conjunto variado de actores, intereses y prácticas sociales concertadas. En esa perspectiva, la governance urbana y territorial aparecería como la capacidad de integrar y dar forma a los distintos intereses y actores, aunque esto no suprime que se planteen desacuerdos o lógicas distintas que influyen en los diversos matices que asume este tipo de acción en el plano regional y territorial (Governa, 2001; Bagnasco y Le Galés, 2000).

La governance puede plantear también distintos problemas en su desarrollo, vinculados a las dificultades para articular lógicas diversas, generar responsabilidades claras de los actores involucrados y disminuir tensiones o conflictos fuertemente arraigados (Stoker, 1998; Rhodes, 1996). Esto destaca, en efecto, la relevancia de considerar tanto los inconvenientes posibles en su implementación como las soluciones en términos de consenso y articulación.

\section{Los pactos territoriales en el escenario italiano reciente}

\subsection{Pactos territoriales y principales elementos del marco institucional italiano}

Desde fines de la Segunda Guerra Mundial hasta principios de los ' 90 , Italia ha atravesado por distintas etapas en lo referente al desarrollo productivo y territorial. En el período comprendido entre los años ' 50 hasta fines de los ' 70 , sobre la base de una política planificada desde el nivel central, las acciones se dirigieron a consolidar una estructura industrial fuerte en los sectores considerados como estratégicos (química, electrónica, automatización industrial, infraestructura de transporte, telecomunicaciones). El actor predominante era la gran empresa fordista ubicada en el denominado "Triángulo Industrial" que conformaban Milán (Lombardía), Turín (Piemonte) y Génova (Liguria) (Tattara y Volpe, 2001 y 2003).

El aumento internacional del petróleo y los problemas en las cuentas públicas determinan para los 
años ' 80 un tipo de política productiva no ya direccionada a sectores específicos, sino al conjunto de las actividades económicas y los diversos territorios. El predominio paulatino de un modelo productivo más flexible o pos-fordista permite el crecimiento espontáneo de pequeñas y medianas empresas (PyMEs) especializadas en bienes tradicionales y localizadas en "distritos industriales" ${ }^{1}$ ubicados fundamentalmente en las regiones del nordeste y centro del país (Véneto, Emilia Romagna, Toscana y Marche). Estas regiones conforman lo que se ha denominado "Tercera Italia", como contraste del noroeste más desarrollado y el sur del país. La política se va focalizando así en el fortalecimiento de ciertas economías externas y los servicios orientados a las pequeñas y medianas empresas (Gurisatti, 2003).

En la década de los noventa el escenario italiano muestra un cambio importante caracterizado por el desarrollo de políticas productivas y territoriales con preponderancia del nivel comunitario (Unión Europea) en los grandes lineamientos orientadores, y la aparición de un nuevo cuadro legislativo de intervención con eje en el plano regional ${ }^{2}$. Con el ingreso

El distrito industrial puede definirse como "una entidad socio-territorial caracterizada por la copresencia activa, en un área territorial circunscripta, natural e históricamente determinada, de una comunidad y de personas y de una población de empresas" (Becattini, 1991). Entre sus principales características podemos citar una elevada división del trabajo entre empresas generalmente de pequeño y mediano porte; procesos de innovación de carácter distributivo; relaciones competitivas/colaborativas de las firmas al interior del distrito; relevancia del territorio (factores socio-culturales) en el apoyo a la creación y difusión del conocimiento y el desarrollo de un conjunto de instituciones formales e informales que regulan la vida económica y dan identidad al sistema local como forma de organización productiva alternativa a la gran empresa (Piore y Sabel, 1984; Brusco, 1990; Becattini, 1998; Bellandi, 1999; Corò, 2002).

La ley 317/1991 para el desarrollo de las pequeñas empresas aparece claramente como la primera intervención de carácter orgánico enteramente dirigida bajo la responsabilidad de las regiones. Es importante aclarar que el caso italiano posee distintos niveles de gobierno además de la administración central: el regional (con 20 regiones); el provincial (con 103 provincias y 2 provincias autónomas: Bolzano y Trento), y el local (con 8.100 comunas). En los últimos años y con distintas legislaciones (leyes No 59/ 1997 y No 112/1998), las regiones han ganado mayor autonomía en términos de planificación y decisiones referidas a las cuestiones del desarrollo. Las provincias también han obtenido particular importancia a nivel legislativo, por de Italia al Euro, el aumento del federalismo (descentralización de funciones en las regiones), la crisis de las finanzas públicas y las fallas en las acciones "asistencialistas" a las áreas más postergadas del sur, comienza a surgir un nuevo tipo de política con clara referencia al "lugar" donde los actores sociales y productivos se desenvuelven.

Esta dimensión obtiene fuerte relevancia en los últimos tiempos como consecuencia de diversos aportes teóricos y trabajos de índole empírico en los diversos países. La cooperación y formación de redes entre actores públicos y privados, la transmisión del conocimiento y la importancia del territorio como base estratégica del desarrollo adquieren una fuerte significación y pasan a ser temas preponderantes en la agenda de política productiva (Porter, 1990; Nonaka y Takeuchi, 1995; Krugman, 1996).

En la segunda mitad de los años ‘ 90 , la política italiana se dirige al estímulo de las prácticas concertadas en el plano territorial haciendo eje en los diferentes sectores productivos. Los índices elevados de desempleo en determinadas regiones o zonas de Europa llevan a que la UE decida destinar una parte de sus Fondos Estructurales a la creación de los primeros pactos territoriales ${ }^{3}$ a favor del empleo y el desarrollo local en zonas con graves problemas socioeconómicos. La UE requiere que los gobiernos regionales y locales se involucren de modo directo en sus territorios y desplieguen políticas innovadoras en términos de empleo y desarrollo productivo. En este sentido, las regiones italianas abandonan progresivamente la asistencia directa sobre la economía a favor de acuerdos negociados con las partes en el ámbito local. El papel que cumplen la denominada "programación negociada" y los "pactos territoriales" adquieren fuerte relevancia.

ejemplo el artículo 13 de la ley No 142/1990 y la norma 19 de la ley No $267 / 2000$ en relación a la planificación espacial del territorio, la valoración del medio ambiente, caminos y transporte. Por otra parte, las comunas adquieren relevancia como la instancia más cercana para estimular procesos productivos consensuados entre los actores y en consonancia con los niveles superiores de gobierno.

3 El concepto de Pacto Territorial fue presentado al Consejo Europeo de Florencia (junio 1996) y respaldado por el Consejo Europeo de Dublín (diciembre de 1996), el cual aprobó un listado de 89 zonas infrarregionales seleccionadas por las administraciones nacionales (González et al., 2005). 
La programación negociada, individualizada con la ley No662/1996, es concebida como un sistema de intervención integral que tiene como objetivo central generar un escenario favorable para el crecimiento económico y la ocupación en un área geográfica determinada. Se parte de la importancia de realizar acciones de relevante complejidad, que involucren diversas competencias institucionales y actores, lo cual requiere, en términos de tiempo, recursos y eficiencia, de la intervención de la correcta cooperación entre el Estado, las regiones y las instancias locales, y de la concertación estratégica con las partes sociales.

Este sistema de actuación supone el encuentro de dos perspectivas en relación a la problemática del desarrollo: a) un enfoque descendente (considerado, top-down), de arriba hacia abajo, y que supone esencialmente la definición y concreción de los objetivos de la programación económica de carácter nacional y sobre todo regional. En este enfoque más tradicional se consideran instrumentos tales como los "convenios institucionales de programa”, los “acuerdos de programa cuadro" y, en algunos aspectos, los "contratos de programa", todos caracterizados por una fuerte predominancia de la administración del Estado en el proceso de definición y negociación ${ }^{4} ; \mathrm{y}$ b) un enfoque ascendente (considerado, bottom-up), de abajo hacia arriba, y que representa la relevancia en términos de actuación de los objetivos del desarrollo local. En esta orientación se destacan como instru-

\footnotetext{
El "convenio institucional de programa" es un arreglo general entre la administración central y regional (o la provincia autónoma), mediante el cual las partes interesadas se proponen colaborar sobre la base de un reconocimiento de los recursos financieros disponibles y los procedimientos administrativos necesarios para la realización de un plan plurianual de intervenciones conjuntas. El "acuerdo de programa cuadro", como derivación del instrumento anterior, plantea que los sujetos del convenio (Estado, Región o Provincia Autónoma), junto con las comunas locales y otros actores públicos y privados, participen en la definición de un programa concreto de acciones de interés común. Esto supone determinar el tipo de intervenciones a realizar, los sujetos responsables involucrados, los recursos disponibles, el monitoreo y control de resultados. Los "contratos de programa”, por su parte, suponen también un acuerdo estipulado en el marco de un "convenio institucional de programa” entre la administración pública y una gran empresa, grupo o consorcio de pequeñas y medianas empresas, de modo de permitir inversiones productivas en áreas delimitadas (pudiendo comprender actividades tales como investigación y servicios).
}

mentos los "contratos de área" y principalmente los "pactos territoriales", caracterizados por ser impulsados por actores públicos y privados a nivel local.

Los pactos territoriales, como uno de los instrumentos más importantes y de mayor extensión de la "programación negociada" en toda Italia, empiezan a desarrollarse a fines de 1996 y se dirigen fundamentalmente a: a) promover la cooperación entre sujetos públicos y privados de un territorio determinado de modo de estimular el diseño y la realización de proyectos de mejoramiento del contexto local (a nivel de industria, servicios e infraestructura); y b) favorecer a través de esos proyectos y por medio de la delimitación territorial y temática un conjunto de inversiones capaces de producir externalidades en términos de ventajas para otras empresas y nuevas inversiones.

La idea de fondo de los pactos territoriales se puede resumir en la intención de favorecer el desarrollo a través de intervenciones que estimulen, con incentivos financieros y servicios, la cooperación de los actores locales (públicos y privados), de modo de poner en funcionamiento proyectos integrados de desarrollo local sometidos a procedimientos de verificación. Se trata entonces de remover los obstáculos que influencian negativamente la productividad, mejorando el contexto en el cual los actores productivos operan o pueden nacer, más que apoyar a la firma individual con incentivos de tipo fiscal o salarial. Así, en un escenario de organización pos-fordista y relevancia del ambiente en el que se desenvuelven los agentes, se busca acrecentar las economías externas tangibles (infraestructuras, servicios) e intangibles (capacidad relacional, capital social), especialmente en las áreas deprimidas del territorio italiano.

Los pactos pueden ser descriptos y analizados a partir de una característica central que es su "horizontalidad", al estimular desde abajo (la comunidad local) y mediante una modalidad de concertación de múltiples actores una programación socioeconómica plurianual. En realidad el instrumento responde a una lógica de promoción de desa-

5 Los "contratos de área" se dirigen a estimular iniciativas empresariales y la creación de nuevos empleos fundamentalmente en áreas industriales con graves crisis de tipo ocupacional y desindustrialización. Los principales sujetos promotores son los sindicatos o los trabajadores mismos. 
rrollo bottom-up con control top-down, ya que prevé la recolección y selección de proyectos de inversión y se concretiza a través de una serie de acuerdos formales entre sujetos de las administraciones estatales, privados y de la sociedad civil.

Los pactos se sustentan en nuevas estrategias de "concertación social" basadas en una metodología claramente local de "lectura del territorio", y donde se valorizan las necesidades y soluciones emanadas por los actores (comunas y otros actores públicos que operan a nivel local, cámaras empresarias, sindicatos, banca, sectores privados, etc.). Con el tiempo la experiencia ha adquirido un significado más amplio y se la consideró como señal de una nueva filosofía de política pública, distinta a la fase de intervención extraordinaria del sur que duró hasta principios de los ' 90 , orientada a generar simultáneamente desarrollo y cohesión social. De esta forma, la introducción en Italia de los principios de una nueva política regional europea ha representado un ulterior empuje a la experimentación de inéditos modelos de política económica mayormente dirigidos a la descentralización de las competencias administrativas en favor de las instituciones intermedias (regiones, comunas, provincias), y la construcción de procesos de governance territorial (Arrighetti y Seravalli, 1999).

Desde este punto de vista, los pactos territoriales responden a dos premisas teóricas fundamentales: a) la idea de "local" como unidad esencial a través de la cual se favorece un desarrollo socioeconómico más general, y b) aquella que, para que se genere desarrollo (local), es necesario implicar desde abajo a todos los actores del territorio mediante un mecanismo de proyección conjunta en grado de producir cohesión y consenso social (Riccone, 2002).

Esto supone un nuevo tipo de política facilitadora de procesos de interacción y partnership local con base en los principios de subsidiariedad, colaboración interinstitucional y competitividad territorial. A diferencia de la típica acción de política de los años '70, que consistía en la provisión de ayudas estatales a las empresas individuales, el eje está puesto en la capacidad de promover agregaciones de intereses e impulsar la innovación en las redes de actores (empresariales, sociales e institucionales) que funcionan en el territorio (Bianchi, 1997; Gainfagna, Rosa y
Sabatini, 2005; Magnatti, Ramella, Trigilia y Viesti, 2005).

La promoción de un pacto territorial, por lo tanto, puede ser impulsada por cualesquiera de los actores locales y supone que sea subscripto por la Región o la Provincia Autónoma (en correspondencia con los programas regionales y criterios de relevancia a nivel de la comunidad), la banca y órganos de financiamiento regionales y de crédito (en el compromiso de financiar y sostener las intervenciones) y la comuna y otros sujetos públicos locales (de modo de acelerar y simplificar los procedimientos e individualizar al responsable de la coordinación y actuación del pacto). Por otra parte, la realización del pacto debe desarrollarse en el lapso de los 48 meses que se establecen a partir de la aprobación oficial.

Los requisitos para activar un pacto están centrados en la concertación entre las partes sociales y en que haya coherencia de la propuesta con los objetivos del desarrollo local de un territorio definido (certificado a través de un convenio protocolizado). $\mathrm{Al}$ mismo tiempo, el pacto territorial debe contener un plan financiero con los compromisos de gasto relativos a cada una de las intervenciones a realizar, indicando los tiempos, la conexión con otros instrumentos de apoyo y las contribuciones como los financiamientos públicos requeridos. Toda esta fase de concertación del pacto viene acompañada por instituciones nacionales que supervisan el desarrollo del proceso (Consejo Nacional de la Economía y el Trabajo, CNEL; Comité Interministerial para la Programación Económica, CIPE).

Focalizando la atención sobre estos aspectos, es posible advertir que, además del sujeto responsable del pacto, se constituye una institucionalidad interna conformada por una pluralidad de organismos (colectivos o individuales, algunos mayormente consultivos y otros con funciones más estrictamente técnicas), como ser los comités ejecutivos representantes de la mesa de concertación, el coordinador institucional, el coordinador y equipo técnico-financiero -representado por el sector público y privado-, el comité comunal y las distintas comisiones desarrolladas a fin de instrumentar las acciones concretas.

Por otra parte, y como plantea Riccone (2002), el pacto territorial ha encontrado particular interés 
en virtud de distintas motivaciones. Un primer motivo se vincula con las características que asume el tipo de "oferta" institucional, dado que el pacto garantizaría importantes recursos para el contexto local, en un marco donde la Comisión Europea (1997), en el ámbito de la modernización de los Fondos Estructurales para el período 2000-2006, ha establecido incentivos financieros y prioridad de asignaciones en territorios con iniciativas de programación concertada ${ }^{6}$.

Una segunda motivación que favoreció la difusión de los pactos puede ser encontrada en razón del estímulo competitivo de los territorios. Como se ha planteado, los procesos de globalización económica han resaltado el ambiente productivo en el cual operan las empresas. En este marco, mediante los pactos territoriales sería posible individualizar de manera puntual los recursos propios de un área geográfica y - a partir de ella- dirigir un conjunto de intervenciones integradas con el objeto de hacer más interesante el entorno productivo elegido. Estas acciones abarcan diversos aspectos, tales como los recursos humanos, infraestructuras, oferta de servicios, desarrollo de sistemas de comunicación, etc. Sobre esta base, los pactos -concebidos como una nueva forma de organización a nivel social y económico- consideran la experiencia de los distritos industriales como modelo en tanto "entidades socioproductivas", en las cuales se registran significativas "economías externas" generadas desde un grado elevado de cooperación e interacción entre las administraciones estatales y el tejido empresarial local.

Las razones de la proliferación de los pactos territoriales, sea tanto del lado de la "oferta" (institucio-

\footnotetext{
6 Los recursos de los Fondos Estructurales para el período de 2000-2006 alcanzan a 195.000 millones de euros y los destinos prioritarios en Italia, España y Francia hasta el presente son: a) regiones menos desarrolladas (objetivo $N^{o} 1$ ). Un PIB por habitante en los tres últimos años inferior al $75 \%$ de la media comunitaria. Obtienen el 69,7\% de los Fondos Estructurales; b) regiones o zonas en proceso de reconversión (objetivo $N^{o} 2$ ). Áreas que sufren transformaciones socioeconómicas profundas (zonas industriales, de servicios, rurales, urbanas y dependientes de la pesca). Alcanzan el 11,5\% del presupuesto de los Fondos Estructurales, y c) apoyo a la adaptación y la modernización de las politicas y sistemas de educación, de formación y de empleo (objetivo $N^{o} 3$ ). Comprenden el $12,3 \%$ del presupuesto de los Fondos Estructurales.
}

nes) como de la "demanda" (mejoramiento del ambiente competitivo), aparecen con claridad para el contexto italiano y llevan a plantear la importancia de estos instrumentos en términos de desempeño y eficacia de las acciones emprendidas. En el apartado siguiente se discutirán con mayor detenimiento estas cuestiones.

\subsection{Pactos territoriales: estado de actuación y eficacia}

Los tiempos de activación de un pacto, su desarrollo en términos de objetivos fijados y cobertura territorial, la rapidez respecto a la utilización de los fondos públicos destinados, el tipo de intervención en el territorio, el nivel de actividades concluidas y el grado de ocupación desarrollado constituyen algunos de los parámetros que miden las condiciones mínimas de buen desempeño de un pacto territorial.

Sobre la base de estas observaciones, el examen de los pactos territoriales puede considerarse tomando en cuenta los principales elementos que caracterizan su universo general, los grados de eficiencia financiera y la eficacia en las acciones desplegadas en 19 casos. Estos aspectos son examinados en relación a los resultados de distintas investigaciones desarrolladas recientemente (CIPE, 2002; Ministerio dell'Economia e delle Finanze e Dipartamento per la Politiche di Sviluppo, 2003; Maganatti, Ramella, Trigilia y Viesti, 2005), donde se indaga sobre las singularidades socioeconómicas e institucionales implicadas en la experiencia de los pactos.

\subsubsection{El universo de los pactos}

La experiencia de los pactos territoriales, desde fines de 1996 hasta principios de 2003, ha llevado a la aprobación de 220 pactos territoriales "nacionales" y 10 europeos. Los pactos fueron aprobados y financiados en bloques sucesivos, cada uno diferente del precedente por reglas de aprobación, gestión y contenido de las intervenciones admitidas. Los pactos de primera generación son los admitidos entre el 18 de diciembre de 1996 y el 26 de junio de 1997. Todos éstos se desarrollan en la zona sur del país y se ponen operativos en los primeros meses de 1998. Los pactos de segunda generación siguen la nueva disciplina introducida por la CIPE a partir del $21 \mathrm{de}$ marzo de 1997. Se ponen operativos en el curso de 1999 y en los primeros meses de 2000. De estos 
Cuadro 1. Pactos por años de activación y financiamiento previsto.

\begin{tabular}{|c|c|c|c|}
\hline Tipos de pacto & Años de activación & Número & $\begin{array}{c}\text { Financiamiento previsto } \\
\text { (en millones) }\end{array}$ \\
\hline \multicolumn{4}{|l|}{ I. Pactos nacionales } \\
\hline . $\quad 1^{\circ}$ generación (a) & 1996 & 12 & 371 \\
\hline . $\quad 2^{\circ}$ generación $(\mathrm{b})$ & 1999 & 39 & 1.287 \\
\hline . Decreto $10 / 10 / 99$ & 2001 & 28 & 1.003 \\
\hline $\begin{array}{l}\text { Instrucción concluida } \\
\text { el } 31 / 12 / 99\end{array}$ & 2001 & 7 & 165 \\
\hline . Agrícolas & 2001 & 91 & 1.386 \\
\hline . Desastres naturales & 2001 & 32 & 902 \\
\hline $\begin{array}{l}\text { Instrucción concluida } \\
\text { el } 28 / 02 / 01\end{array}$ & $\begin{array}{l}\text { A ser financiados } \\
\text { en el } 2003\end{array}$ & 11 & 440 \\
\hline Total & & 220 & 5.545 \\
\hline II. Pactos Europeos & 1998 & 10 & 381 \\
\hline Total & & 230 & 5.926 \\
\hline
\end{tabular}

Fuente: Ministerio dell'Economia, Dipartamento per la Politiche di Sviluppo e di Coesione (2003). Nota. Por "año de activación" se entiende el año de autorización y erogación de los fondos.

pactos 20 se desarrollan en las regiones del sur y 19 en el centro-nordeste del país. Así, los pactos pueden dividirse temáticamente según su importancia en pactos generales $\left(1^{\circ} \mathrm{y} 2^{\circ}\right.$ generación, por tipo de instrucción y decreto, pactos europeos) y agrícolas (Cuadro 1).

En lo referente a la cobertura demográfica y territorial de los pactos, es posible advertir la fuerte importancia que alcanza el sur del país (con alrededor del $80 \%$ de la población y superficie cubierta por este tipo de instrumento). En el centro-nordeste esta proporción es de alrededor de un 30\% del total de habitantes y de extensión territorial (Cuadro 2).

Si se consideran algunas categorías de pactos la destinación al sur es fuertemente predominante; en otros casos, sobre todo para los pactos, de segunda generación y los de territorios afectados por desastres naturales, la presencia del centro-nordeste es significativa. En este sentido, las regiones que más pactos han activado hasta la actualidad son Sicilia (49), Campania (30), Puglia (26), y al centro-nordeste, Piemonte (17) y Véneto (14) (Ministerio dell'Economia-Dipartamento per la Politiche di Sviluppo e di Cohesione, 2005; ver anexo).

Finalmente, si se considera el impacto ocupacional de los pactos sobre la base de distintos análisis estimados, es posible advertir un incremento de alrededor de un $40 \%$ en los niveles de empleo. En este sentido, los avances en ocupación más significativos estarían focalizados en los pactos de $1^{a}$ genera- ción, agrícolas y los más recientes (de 2001 en adelante).

\subsubsection{Eficiencia financiera}

Los datos disponibles sobre la eficiencia financiera del instrumento toman en cuenta los siguientes aspectos: a) los niveles de activación en cuanto las iniciativas financiadas; b) las proporciones de la erogación; c) la velocidad de gasto.

Las cuestiones vinculadas con la activación reflejan una fuerte variedad entre los pactos, habiendo situaciones de notable avance junto a otras con graves demoras, dándose esto inclusive para los pactos con elevada antigüedad. Aparece como un hecho muy marcado el que más de la tercera parte de los pactos no ha tenido iniciativas activadas; como medianamente positiva, la situación de los pactos europeos, y respecto a la fecha de activación, la situación de los pactos del decreto 10 de octubre de 1999 (Cuadro 3).

El estado de actuación de los pactos se ve favorecido también por la información referida a las proporciones de erogación o gasto sobre el total de recursos estipulados. Estos datos muestran coherencia con aquellos vinculados a los de activación. Con la excepción de los pactos europeos, es importante el número de pactos con erogación inferior (casi la mitad del total), con menos de $1 / 3$ de las erogaciones desarrolladas, y sin erogación. De la misma forma, con más de $2 / 3$ de erogación se encuentran solamente 8 pactos europeos cofinanciados y 1 pacto de primera generación (Cuadro 4). 
Cuadro 2. Cobertura demográfica y territorial de los pactos por región.

\begin{tabular}{|c|c|c|c|c|c|c|}
\hline \multirow[b]{2}{*}{ Región } & \multicolumn{3}{|c|}{ Población } & \multicolumn{2}{|r|}{ Superficie } & \multirow{2}{*}{$\begin{array}{c}(\mathrm{Km}) \\
\mathrm{C} / \mathrm{D} \\
\%\end{array}$} \\
\hline & $\begin{array}{c}\text { Población } \\
\text { de las } \\
\text { comunas } \\
\text { partícipes } \\
\text { en pactos (A) }\end{array}$ & $\begin{array}{l}\text { Población } \\
\text { total (B) }\end{array}$ & $\begin{array}{c}\mathrm{A} / \mathrm{B} \\
\%\end{array}$ & $\begin{array}{l}\text { Población de } \\
\text { las comunas } \\
\text { partícipes } \\
\text { en pactos } \\
\text { (C) }\end{array}$ & $\begin{array}{l}\text { Superficie } \\
\text { total (D) }\end{array}$ & \\
\hline Piemonte & 839.712 & 4.289 .731 & 19,57 & 6.842 & 24.816 & 27,57 \\
\hline Valle d'Aost & & 120.589 & 0,00 & & 3.224 & 0,00 \\
\hline Lombardía & 400.145 & 7.820 .164 & 5,12 & 460 & 23.099 & 1,99 \\
\hline Trentino-Alto Adige & 1.457 & 943.123 & 0,15 & 9 & 13.435 & 0,07 \\
\hline Véneto & 1.311 .530 & 4.540 .853 & 28,88 & 7.155 & 18.097 & 39,54 \\
\hline Friuli-Venezia Giulia & 56.819 & 1.188 .594 & 4,78 & 400 & 7.743 & 5,17 \\
\hline Liguria & 1.342 .884 & 1.621 .016 & 82,84 & 3.851 & 5.304 & 72,61 \\
\hline Emilia-Romagna & 477.665 & 4.008 .663 & 11,92 & 6.902 & 21.960 & 31,43 \\
\hline Toscana & 1.803 .372 & 3.547 .604 & 50,83 & 15.940 & 22.850 & 69,76 \\
\hline Umbría & 651.873 & 840.482 & 77,56 & 5.836 & 8.410 & 69,39 \\
\hline Marche & 871.321 & 1.469 .195 & 59,31 & 7.355 & 9.567 & 76,88 \\
\hline Lazio & 1.181 .126 & 2.646 .334 & 44,63 & 5.059 & 17.030 & 29,71 \\
\hline Total Centro-Nordeste & 8.937 .904 & 33.036 .348 & 27,05 & 59.809 & 175.535 & 34,07 \\
\hline Abruzzo & 690.004 & 1.281 .283 & 53,85 & 6.617 & 10.651 & 62,13 \\
\hline Molise & 230.606 & 327.177 & 70,48 & 3.091 & 4.366 & 70,80 \\
\hline Campania & 3.167 .927 & 4.781 .775 & 66,25 & 11.322 & 13.320 & 85,00 \\
\hline Puglia & 3.971 .833 & 4.086 .608 & 97,19 & 18.873 & 19.235 & 98,12 \\
\hline Basilicata & 555.334 & 604.807 & 91,82 & 8.709 & 9.926 & 87,74 \\
\hline Calabria & 1.269 .348 & 2.043 .288 & 62,12 & 9.220 & 14.870 & 62,00 \\
\hline Sicilia & 4.836 .070 & 5.076 .700 & 95,26 & 24.890 & 25.516 & 97,55 \\
\hline Cerdeña & 1.188 .585 & 1.648 .044 & 72,12 & 13.964 & 23.892 & 58,45 \\
\hline Total sur & 15.909 .707 & 19.849 .682 & 80,15 & 96.686 & 121.776 & 79,40 \\
\hline Total Italia & 24.847 .611 & 52.886 .030 & 46,98 & 156.495 & 297.311 & 52,64 \\
\hline
\end{tabular}

Fuente: Ministerio dell'Economia, Dipartamento per la Politiche di Sviluppo e di Coesione (2003).

Cuadro 3. Pactos por cantidad de iniciativas empresariales activadas (30-6-02).

\begin{tabular}{|c|c|c|c|c|c|}
\hline \multirow[t]{2}{*}{ Tipos de Pacto } & Cantidad de & iniciativas & activadas & \multirow{2}{*}{$\begin{array}{l}\text { Pactos sin } \\
\text { iniciativas } \\
\text { activadas }\end{array}$} & \multirow[t]{2}{*}{ Total } \\
\hline & $\begin{array}{l}\text { Inferior } \\
\text { a } 1 / 3 \\
\end{array}$ & $\begin{array}{c}\text { Entre } 1 / 3 \\
\text { y } 2 / 3 \\
\end{array}$ & $\begin{array}{c}\text { Más de } \\
2 / 3 \\
\end{array}$ & & \\
\hline \multicolumn{6}{|l|}{ I. Pactos nacionales } \\
\hline . $1^{\circ}$ generación & 2 & 5 & 5 & 0 & 12 \\
\hline . $2^{\circ}$ generación & 5 & 20 & 14 & 0 & 39 \\
\hline - Decreto 10/10/99 & 9 & 7 & 10 & 2 & 28 \\
\hline $\begin{array}{l}\text { Instrucción concluida } \\
\text { el } 31 / 12 / 99 \\
\end{array}$ & 4 & 0 & 0 & 3 & 7 \\
\hline . Agrícolas & 34 & 9 & 4 & 44 & 91 \\
\hline . Desastres naturales & 9 & 0 & 0 & 23 & 32 \\
\hline $\begin{array}{l}\text {. Instrucción concluida } \\
\text { el } 28 / 02 / 01\end{array}$ & 0 & 0 & 0 & 11 & 11 \\
\hline Total & 63 & 41 & 33 & 83 & 220 \\
\hline II. Pactos Europeos & 1 & 1 & 8 & 0 & 10 \\
\hline$T$ & & & & & \\
\hline Total & 64 & 42 & 41 & 83 & 230 \\
\hline
\end{tabular}

Fuente: Ministerio dell'Economia, Dipartamento per la Politiche di Sviluppo e di Coesione (2003). 
La velocidad de erogación, comprendida como la relación entre los recursos erogados y tiempo del curso de activación, muestra en casi el $60 \%$ de los pactos un nivel muy bajo (inferior al 10\%). Los pactos europeos se distinguen en relación al resto por los buenos niveles alcanzados ( 8 de 10 pactos superan el 30\% de los recursos erogados desde el tiempo de su activación) (Cuadro 5). Por parte, es posible advertir que la velocidad de erogación es mayor en los pactos localizados en el sur; dado la intensidad e importancia de la ayudas suministradas (CIPE, 2002).

La atención puesta en estos aspectos hace lugar a la relevancia de nuevos instrumentos de política e incentivos orientados a relanzar y estimular la competitividad de los territorios desde una perspectiva de control riguroso de los recursos erogados (con fuerte anclaje a nivel de las regiones), en un escenario europeo caracterizado por el cumplimiento estricto de metas fiscales por parte de las naciones miembro (Gainfagna, Rosa y Sabatini, 2005).

\subsubsection{Eficacia de los pactos}

En este apartado, el objetivo está puesto en analizar la experiencia de 19 pactos (5 en el centronordeste y 14 en el sur) que han estado operativos por al menos tres años y que han tenido un buen desempeño en base a los criterios examinados ante-

Cuadro 4. Pactos según cantidad de recursos erogados (30-6-02).

\begin{tabular}{|c|c|c|c|c|c|}
\hline \multirow[t]{2}{*}{ Tipos de Pacto } & \multicolumn{3}{|c|}{ Cantidad de erogaciones desarrolladas } & \multirow{2}{*}{$\begin{array}{l}\text { Pactos sin } \\
\text { erogaciones }\end{array}$} & \multirow[t]{2}{*}{ Total } \\
\hline & $\begin{array}{c}\text { Inferior } \\
\text { a } 1 / 3 \\
\end{array}$ & $\begin{array}{c}\text { Entre } 1 / 3 \\
\text { y } 2 / 3 \\
\end{array}$ & $\begin{array}{c}\text { Más de } \\
2 / 3 \\
\end{array}$ & & \\
\hline \multicolumn{6}{|l|}{ I. Pactos nacionales } \\
\hline$\cdot 1^{\circ}$ generación & 4 & 7 & 1 & 0 & 12 \\
\hline$\cdot 2^{\circ}$ generación & 24 & 15 & 0 & 0 & 39 \\
\hline - Decreto $10 / 10 / 99$ & 23 & 3 & 0 & 2 & 28 \\
\hline \begin{tabular}{|c|}
. Instrucción concluida \\
el $31 / 12 / 99$ \\
\end{tabular} & 4 & 0 & 0 & 3 & 7 \\
\hline - Agrícolas & 44 & 3 & 0 & 44 & 91 \\
\hline . Desastres naturales & 13 & 0 & 0 & 19 & 32 \\
\hline $\begin{array}{l}\text {. Instrucción concluida } \\
\text { el } 28 / 02 / 01\end{array}$ & 0 & 0 & 0 & 11 & 11 \\
\hline Total & 112 & 28 & 1 & 79 & 220 \\
\hline II. Pactos Europeos & 1 & 1 & 8 & 0 & 10 \\
\hline Total & 113 & 29 & 9 & 79 & 230 \\
\hline
\end{tabular}

Fuente: Ministerio dell'Economia, Dipartamento per la Politiche di Sviluppo e Coesione (2003).

Cuadro 5. Pactos por velocidad de erogación.

\begin{tabular}{|c|c|c|c|c|}
\hline \multirow[t]{2}{*}{ Tipos de Pacto } & \multicolumn{3}{|c|}{ Velocidad de erogación } & \multirow[t]{2}{*}{ Total } \\
\hline & Inferior a $10 \%$ & $\begin{array}{c}\text { Entre el } 10 \\
\text { y el } 30 \%\end{array}$ & $\begin{array}{c}\text { Más del } \\
30 \%\end{array}$ & \\
\hline \multicolumn{5}{|l|}{ I. Pactos nacionales } \\
\hline$\cdot 1^{\circ}$ generación & 4 & 8 & 0 & 12 \\
\hline$\cdot 2^{\circ}$ generación & 19 & 20 & 0 & 39 \\
\hline - Decreto $10 / 10 / 99$ & 5 & 20 & 3 & 28 \\
\hline $\begin{array}{l}\text { - Instrucción concluida } \\
\text { el } 31 / 12 / 99\end{array}$ & 4 & 3 & 0 & 7 \\
\hline -Agrícolas & 59 & 20 & 12 & 91 \\
\hline - Desastres naturales & 28 & 3 & 1 & 32 \\
\hline $\begin{array}{l}\text { - Instrucción concluida } \\
\text { el } 28 / 02 / 01\end{array}$ & 11 & 0 & 0 & 11 \\
\hline Total & 130 & 74 & 16 & 220 \\
\hline II. Pactos Europeos & 2 & 0 & 8 & 10 \\
\hline Total & 132 & 51 & 47 & 230 \\
\hline
\end{tabular}

Fuente: Ministerio dell'Economia, Dipartamento per la Politiche di Sviluppo e Coesione (2003). 
Los pactos territoriales en Italia. Un análisis de la experiencia reciente y sus implicancias en términos de governance

Cuadro 6. Tipología de las intervenciones y de las infraestructuras públicas.

\begin{tabular}{|c|c|c|}
\hline Tipo de intervención & Número de proyectos & $\%$ \\
\hline - Viabilidad (calles, transporte, etc) & 32 & 22,4 \\
\hline - Centros de congresos y ferias de exposición & 6 & 4,2 \\
\hline $\begin{array}{l}\text { Áreas industriales (construcción, urbanización y } \\
\text { ampliación de espacios para el desarrollo de actividades } \\
\text { industriales) }\end{array}$ & 17 & 11,9 \\
\hline $\begin{array}{l}\text { Potenciación de obras hídricas, eléctricas, plantas de depuración } \\
\text { y tratamiento de desechos }\end{array}$ & 13 & 9,1 \\
\hline $\begin{array}{l}\text { Valorización de los recursos del territorio (museos, áreas } \\
\text { arqueológicas, recuperación de centros históricos, restauración } \\
\text { de monumentos y edificios, realización de itinerarios turísticos } \\
\text { y recorridos naturales, valorización de los parques naturales, } \\
\text { construcción de parques temáticos, preparación de centros } \\
\text { de información y acomodación turística, etc.). }\end{array}$ & 60 & 42,0 \\
\hline $\begin{array}{l}\text { Otras intervenciones (realización de estructuras deportivas, obras } \\
\text { de urbanización, recuperación de edificios y establecimientos } \\
\text { industriales) }\end{array}$ & 15 & 10,5 \\
\hline Total & 143 & 100,0 \\
\hline
\end{tabular}

Fuente: Ministerio dell'Economia, Dipartamento per la Politiche di Sviluppo e di Coesione (2003).

riormente (Ministerio dell'Economia, Dipartamento per la Politiche di Sviluppo e di Coesione, 2003). La idea está puesta en considerar la eficacia de los pactos en términos de su impacto económico e institucional, tomando en cuenta la utilización de los recursos públicos y locales en función del empleo y unidades productivas generadas, el desarrollo de infraestructura y bienes colectivos ${ }^{7}$, la formación de governance local y el mejoramiento de la estructura productiva y de la situación local en su conjunto.

En líneas generales, los 19 pactos analizados han recibido una contribución estatal (y de la UE) cercana a los 780 millones de euros y han generado alrededor de 15 mil puestos de trabajo. Esto se suma a la movilización, a nivel local, de otros 920 millones de euros correspondientes a cerca de 1.800 iniciativas proyectadas. Un $45 \%$ de los 1.400 proyectos empresariales financiados con contribuciones públicas son nuevas unidades productivas (630 empresas).

Los "bienes colectivos" hacen lugar a los bienes y servicios imposibles de ser divididos y cuyo disfrute está referido a la pluralidad de individuos y no meramente a un sujeto en particular.
Es importante destacar que los pactos territoriales no pueden ser sólo evaluados teniendo en cuenta su performance financiera. Uno de los objetivos en el despliegue de este tipo de instrumentos está centrado en generar un cambio positivo del contexto socioinstitucional en el cual operan las empresas y los ciudadanos desde una perspectiva participativa y consensuada. La importancia está puesta, por tanto, en favorecer el mejoramiento de la governance y la calidad de vida de la comunidad local, incrementando la dotación de bienes colectivos y potenciando el capital social tanto en el plano institucional como de los actores participantes.

En los 19 pactos examinados se prevén iniciativas infraestructurales a partir de 143 proyectos dirigidos a aumentar directa o indirectamente la dotación territorial de bienes y servicios colectivos. La mayor parte de estos proyectos se dirige a la valorización de los recursos del territorio en el plano de intervenciones sobre el patrimonio histórico-arquitectónico, el medio ambiente y la estructura turística (Cuadro 6).

En este contexto, se destaca, entre otras experiencias, la del Pacto Territorial de Sangro Aventino de la Región de Abruzzo, que abarca 13 municipios de la provincia de Aquila y 46 municipios de la 
provincia de Chieti. El Pacto ha sido aprobado por decisión de la Comisión de la UE y cofinanciado por deliberación CIPE de julio de 1998, llegando las inversiones a 125,19 millones de euros (73,53 millones de contribuciones a cargo de los privados y 51,65 de contribuciones públicas) y una ocupación estimada en 1.222 puestos de trabajo. La mayor parte de las iniciativas se han concentrado en fortalecer el sistema productivo local, propiciar la creación y/o mejoramiento de áreas para el turismo, desplegar acciones de mejora del medio ambiente y apoyar el desarrollo de los recursos humanos (Cuadro 7).

Por otra parte, en base a la percepción relevada mediante distintas entrevistas a los actores implicados de una u otra forma en los distintos pactos (empresarios, técnicos, políticos y funcionarios públicos, representantes de cámaras empresarias y sindicatos, etc.), es posible advertir un fuerte reconocimiento sobre el impacto que tiene este tipo de instrumentos en los diversos territorios. Los 19 pactos serían medianamente eficaces en el mejoramiento del contexto local, pero esto varía según el tipo de impacto considerado: el juicio es altamente positivo en las cuestiones que conciernen a la governance local y el sistema de relaciones entre los actores; es positivo también en lo relativo a la estructura económica; es más bajo en cuanto al desarrollo de bienes colectivos, especialmente en relación al desarrollo de infraestructuras sociales (Cuadro 8).

En este sentido, los distintos pactos analizados muestran grados de variabilidad en su performance a nivel económico e institucional. Esto resalta la complejidad que se desarrolla al interior de los pactos, donde la sola obtención de eficiencia financiera no constituiría condición suficiente para su eficacia. Así, es posible advertir que la performance de los pactos de primera generación y europeos son superiores a aquellos de los de segunda generación (eso viene interpretado como una mayor atención prestada en los primeros a la construcción gradual de relaciones de cooperación entre los sujetos). Por otra parte, los pactos realizados en áreas más atrasadas no muestran grandes diferencias de desempeño respecto a los de territorios más avanzados.

Esto estaría demostrando que en el buen desempeño de los pactos, más que influir las condiciones de disponibilidad de recursos, serían fundamentales cuestiones tales como el contexto institucional, el liderazgo (público o privado) en la movilización del proceso y el grado de cohesión e interacción que se establece entre los distintos actores involucrados. Al mismo tiempo, los problemas en la selección de prioridades, la falta de coordinación institucional y las debilidades en la implementación de las acciones se presentan como los obstáculos más frecuentes de este tipo de instrumentos (Mele, 2000; Paola, 2001).

La realización de un análisis cuidadoso de las potencialidades y recursos del territorio, el desarrollo de concertaciones donde los actores compartan el proyecto y los objetivos, la selección coherente de las inversiones y la creación de externalidades positivas aparecen como factores centrales a la hora de evaluar las experiencias de mejor desempeño (Di Meglio, 2005).

\section{Consideraciones finales}

Los pactos territoriales italianos han constituido una experiencia original de política basada en un proyecto colectivo (negociado y concertado) de de-

Cuadro 7. Inversiones del Pacto Sangro Aventino según área de intervención en millones de euros.

\begin{tabular}{|l|c|c|c|}
\hline Área de intervención & Número de iniciativas & Inversión facilitada & Contribución pública \\
\hline Sistema productivo local & 97 & 64,94 & 21,13 \\
\hline Turismo y ambiente & 95 & 41,51 & 17,18 \\
\hline Agricultura & 20 & 4,42 & 2,20 \\
\hline $\begin{array}{l}\text { Infraestructuras de soporte } \\
\text { al desarrollo local }\end{array}$ & 9 & 8,43 & 5,65 \\
\hline Recursos humanos & 84 & 3,11 & 3,11 \\
\hline $\begin{array}{l}\text { Mejoramiento de las } \\
\text { condiciones de vida }\end{array}$ & 7 & 1,42 & 1,00 \\
\hline Actuación del Pacto territorial & 1 & 1,37 & 1,37 \\
\hline Total & $\mathbf{3 1 3}$ & $\mathbf{1 2 5 , 1 9}$ & 51,65 \\
\hline
\end{tabular}

Fuente: Giunta Regionale Abruzzo. 
Los pactos territoriales en Italia. Un análisis de la experiencia reciente y sus implicancias en términos de governance

Cuadro 8. Nivel de eficacia percibida por los actores del Pacto en cuanto al mejoramiento de la situación local (en \%).

\begin{tabular}{|c|c|}
\hline Governance local & 84,3 \\
\hline - Relaciones de confianza entre las organizaciones de relevancia colectiva & 79,3 \\
\hline - Relaciones de confianza entre los actores privados y las instancias gubernamentales & 85,3 \\
\hline - Discusión de ideas guías para promover el desarrollo del territorio & 91,4 \\
\hline - Relaciones con los sindicatos & 75,9 \\
\hline \multicolumn{2}{|l|}{ - Agilización y funcionalidad de los procedimientos administrativos para las } \\
\hline actividades económicas & 88,8 \\
\hline \multicolumn{2}{|l|}{ - Capacidad de proyección de las administraciones locales y de promoción } \\
\hline del desarrollo local & 85,3 \\
\hline Bienes colectivos y atracción del territorio & 69,6 \\
\hline - Fomación de la fuerza de trabajo & 71,6 \\
\hline - Dotación de servicios/infraestructura colectiva para las empresas & 77,6 \\
\hline - Atracción del territorio para las inversiones externas & 76,7 \\
\hline - Dotación de infraestructura social & 52,6 \\
\hline Estructura económica & 81,5 \\
\hline - Capacidad innovativa de las empresas & 94,8 \\
\hline - Calidad de los productos & 85,3 \\
\hline - Cooperación entre las empresas & 72,4 \\
\hline - Relaciones de confianza entre las empresas & 73,3 \\
\hline Mejoramiento del conjunto & 78,5 \\
\hline
\end{tabular}

Fuente: Ministerio dell'Economia, Dipartamento per la Politiche di Sviluppo e di Coesione (2003).

sarrollo y de transformación de la economía y la sociedad local. La importancia puesta en generar nuevas formas de governance territorial y estimular procesos de diálogo y cooperación entre los actores para producir mecanismos "generativos" en las distintas comunidades aparecen como los grandes aciertos de este tipo metodología de acción.

Los resultados obtenidos en relación a la eficiencia y eficacia de los pactos territoriales indican claramente que este tipo de instrumento no ha dado respuestas unívocas. La experiencia muestra desempeños favorables tanto en zonas atrasadas como en los territorios más prósperos. En este escenario, distintos elementos aparecen como importantes a la hora de evaluar a los pactos: la formación de nuevas dirigencias y liderazgos -en el plano político, empresarial y social-capaces de movilizar a los territorios, el desarrollo de nuevas inversiones e infraestructuras y el despliegue de mecanismos de concertación entre iniciativas públicas y privadas para favorecer la transformación efectiva del contexto socioeconómico e institucional en el cual actúan las empresas y los ciudadanos.

En términos generales, se puede señalar que en gran parte de los casos las iniciativas contribuyeron a dibujar un entramado institucional y social más consistente y articulado, caracterizado por importantes niveles de participación y consenso. Esta metodología organizada desde "abajo" y con fuerte control institucional nacional y regional de los recursos permitió el tratamiento de proyectos de naturaleza compleja (planificación urbanística, proyección socioeconómica del territorio, etc.) y la intervención de una multiplicidad de actores (comunas y distintos niveles de la administración gubernamental, cámaras empresarias, sindicatos, banca, sectores privados, etc.), a fin de establecer una estrategia integrada y colectiva de promoción del territorio.

En el caso de América Latina, la experiencia de los pactos puede ser válida para pensar nuevos escenarios de política que tengan en cuenta las particularidades de los territorios y el apoyo al desarrollo local desde una estrategia de coordinación multinivel y la incorporación de un número amplio de actores en el análisis de las decisiones. Esto supone, por un lado, valorizar la importancia del desarrollo local como una política que no sólo incumbe a los gobiernos locales, sino que requiere de la cooperación, asistencia técnica, recursos y control a lo largo del tiempo de los otros niveles de gobierno (nacional, regional o provincial, y supranacional de ser posible). Por otro lado, 
plantea la necesidad de una verdadera concertación en el plano territorial, donde los actores públicos, privados y sociales comportan los objetivos, prioricen los mejores proyectos y lleven a cabo las acciones pertinentes en el corto y mediano plazo.

En este sentido, los pactos territoriales italianos constituyen actualmente un potente instrumento en términos de governance, que con sus particularidades pueden ser adaptados en nuestros países de modo de hacer más eficaces y legítimas las políticas, y estimular, mediante la movilización de una gran diversidad de actores y niveles de gobierno, una nueva modalidad de acción colectiva a nivel local.

\section{Referencias bibliográficas}

Amin, A. (1999). "An institutionalist perspective on regional economic development". International Journal of Urban and Regional Research, 23, 2: 365-378.

Arrighetti, A. y G. Saravalli (eds.) (1999). Istituzioni intermedie e sviluppo locale. Roma: Donzelli.

Bagnasco, A. y P. le Galés (comps.) (2000). Cities in contemporary Europe. Cambridge: Cambridge University Press.

Beccatini, G. (1991). "Il distretto industriale marshalliano come concetto socio-economico". Pyke, F., G. Becattini y W. Sengerberger (eds.), Distretti industriali e corporazione fra le imprese. Studi e informazioni. Banca Toscana Quaderni, 34: 197-231.

Bellandi, M. (1999). "Terza Italia e distretti industriali”. AAVV, L'Industria, storia d'Italia. Annali, 15. Torino: Einaudi.

Bennett, R. J. y G. Krebs (1994). "Local economic development partnership: an analysis of policy networks in EC-LEDA local employment strategies". Regional Studies, 28, 2: 119-140.

Bianchi, P. (1997). Construir el mercado. Lecciones de la Unión Europea: el desarrollo de las instituciones $y$ de las políticas de competitividad. Buenos Aires: Universidad Nacional de Quilmes.

Boscherini, F. y L. Poma (2000). "Más allá de los sistemas industriales: El nuevo concepto del territorio en el marco de la economía global". Boscherini, F. y L. Poma (comps.), Territorio, conocimiento y competitividad de la empresa: El rol de las instituciones en el espacio global. Buenos Aires/Madrid: Miño y Dávila Editores, 23-38.
Brugué, Q., R. Goma y J. Subirats (2002). “Gobierno y territorio: del Estado a las redes". Subirats, J. (comp.), Redes, territorios y gobierno. Nuevas respuestas locales a los restos de la globalización. Barcelona: Diputación Barcelona, Xarxa de Municipios, 299-310.

Brusco, S. (1990). "The idea of the industrial district: its genesis". Pyke, F., G. Becattini y W. Sengerberger (comps.), Industrial districs and inter-firm co-operation in Italy. Geneva: ILO.

CIPE (2002). Patti territoriali: alcuni elementi appresi del monitoraggio e dalle esperienze di valutazione. Unità di valutazione del Dipartimento per le Politiche di Sviluppo e di Cohesione.

Corò, G. (2002). "Le politiche per i distretti industriali da strumento 'speciale' a occasione per una nuova politica economica regionale”. Economia e Società Regionale, 5.

Courlet, C. y B. Soulage (1995). "Industrial dynamics and territorial space". Entrepreneurship \& Regional Development, 7: 287-307.

Di Meglio, R. (2005). Los pactos territoriales en la experiencia italiana. www.redelaldia.org.

Faludi, A. (2005): "La política de cohesión territorial de la Unión Europea”, Boletín de la A.G.E. No39-2005, págs. 11-30.

González, R. L. et al. (2005). "Los pactos territoriales a favor del empleo en España”. Boletín de la A.G.E., 39: 335-360.

Gainfagna, A., G. Rosa y M. Sabatini, M. (2005). Rapporto sul processo di riforma degli incentivi nel Mezzogiorno: La percezione delle parti socio economiche, CNEL.

Governa, F. (2001). "Del goverment a la governance. El canvi de les formes i les modalitats de láccio colectiva en el camp urbà i territorial”. Documents d'Anàlisi Geogràfica, 41: 43-62.

Gurisatti, P. (2003). Una nuova dimensione e un nuovo paradigma per la Politica Industriale (mimeo).

Krugman, P. (1996). Geography and trade. Gaston Eyskens Lecture.

Mayntz, R. (2001): "El Estado y la sociedad civil en la gobernanza moderna". Reformay Democracia, 21.

Maganatti, P. F. Ramella, C. Trigilia y G. Viesti (2005). Patti territoriali. Lezioni per lo sviluppo. Bologna: Il Mulino-Ricerca.

Mele, G. (2000). Ruolo della partnership e aspettative delle imprese: una prospettiva più realistica per $i$ PIT. COFINDUSTRIA. 
Ministerio dell'Economia e delle Finanze, Dipartamento per la Politiche di Sviluppo e di Coesione (2003). La lezione dei Patti territoriali per la progettazione integrata territoriale nel Mezzogiorno. Ministerio dell'Economia e delle Finanze e Dipartamento per la Politiche di Sviluppo e di Coesione.

(2005). Rapporto

Annuale del DPS. Ministerio dell'Economia e delle Finanze e Dipartamento per la Politiche di Sviluppo e di Coesione.

Nonaka, I. y H. Takeuchi (1995). The knowledgecreating company. New York: Oxford University Press.

Paola, I. (2001). Profili problematici del Patto Territoriale. www.unicz.it/lavoro/lavoro.htm.

Pierre, J. (ed.) (2000). Debating governance. Oxford: Oxford University Press.

Piore, M. y C. Sabel (1984). The second industrial divide. New York: Basic Books.

Porter, M. (1990). The competitive advantage of nations. New York: The Free Press.
Riccone, P. (2002). "Programmazione negoziata e sviluppo locale: tre patti territoriali a confronto". Rivista Italiana di Politiche Pubbliche, 2: 89198.

Rhodes, R. A. W. (1996). "The New Governance: Governing without government”. Political Studies, 44.

Stoker, G. (1998). "El buen gobierno como teoría: cinco propuestas". Revista Internacional de Ciencias Sociales, 155.

Tattara, G. y Volpe, M. (2001). "I distretti industriale definizioni e storia”. Tattara, G. (ed.), Il piccolo che nasce dal grande. Le cento facce dei distretti industriali. Milano: Franco Angeli.

(2003). "Las redes en los

distritos industriales italianos: la Terza Italia". Boscherini, F., M. Novick y G. Yoguel (comps.), Nuevas tecnologías de información y comunicación. Los límites en la economía del conocimiento. Buenos Aires: Miño y Dávila/UNGS. 
Rodrigo Carmona

\section{Anexo}

1. Pactos territoriales italianos activados por región y tipo hasta el 31 de diciembre de 2005.

\begin{tabular}{|c|c|c|}
\hline Regiones & Tipo de pacto & No de activados \\
\hline \multirow[t]{3}{*}{ Piemonte } & - generales & 11 \\
\hline & - agrícolas & 6 \\
\hline & - total & 17 \\
\hline \multirow[t]{3}{*}{ Lombardía } & - generales & 1 \\
\hline & - agrícolas & - \\
\hline & - total & 1 \\
\hline \multirow[t]{3}{*}{ Liguria } & - generales & 4 \\
\hline & - agrícolas & 2 \\
\hline & - total & 6 \\
\hline \multirow[t]{3}{*}{ Véneto } & - generales & 9 \\
\hline & - agrícolas & 5 \\
\hline & - total & 14 \\
\hline \multirow[t]{3}{*}{ Friuli Venezia Giulia } & - generales & 1 \\
\hline & - agrícolas & - \\
\hline & - total & 1 \\
\hline \multirow[t]{3}{*}{ Emilia Romagna } & - generales & 3 \\
\hline & - agrícolas & 1 \\
\hline & - total & 4 \\
\hline \multirow[t]{3}{*}{ Toscana } & - generales & 7 \\
\hline & - agrícolas & 6 \\
\hline & - total & 13 \\
\hline \multirow[t]{3}{*}{ Umbría } & - generales & - \\
\hline & - agrícolas & 2 \\
\hline & - total & 2 \\
\hline \multirow[t]{3}{*}{ Marche } & - generales & 2 \\
\hline & - agrícolas & 2 \\
\hline & - total & 4 \\
\hline \multirow[t]{3}{*}{ Lazio } & - generales & 5 \\
\hline & - agrícolas & - \\
\hline & - total & 5 \\
\hline \multirow[t]{3}{*}{ Abruzzo } & - generales & 4 \\
\hline & - agrícolas & - \\
\hline & - total & 4 \\
\hline \multirow[t]{3}{*}{ Molise } & - generales & - \\
\hline & - agrícolas & 2 \\
\hline & - total & 2 \\
\hline \multirow[t]{3}{*}{ Campania } & - generales & 14 \\
\hline & - agrícolas & 16 \\
\hline & - total & 30 \\
\hline \multirow[t]{3}{*}{ Puglia } & - generales & 15 \\
\hline & - agrícolas & 11 \\
\hline & - total & 26 \\
\hline \multirow[t]{3}{*}{ Basilicata } & - generales & 4 \\
\hline & - agrícolas & 1 \\
\hline & - total & 5 \\
\hline \multirow[t]{3}{*}{ Calabria } & - generales & 12 \\
\hline & - agrícolas & 3 \\
\hline & - total & 15 \\
\hline \multirow[t]{3}{*}{ Sicilia } & - generales & 24 \\
\hline & - agrícolas & 25 \\
\hline & - total & 49 \\
\hline \multirow[t]{3}{*}{ Cerdeña } & - generales & 6 \\
\hline & - agrícolas & 9 \\
\hline & - total & 15 \\
\hline
\end{tabular}

Fuente: Ministero dell'Economia, Dipartimento Politiche di Sviluppo e di Coesione (2005). 
Los pactos territoriales en Italia. Un análisis de la experiencia reciente y sus implicancias en términos de governance

\section{Listado de los 19 pactos terrritoriales analizados}

Benevento

Caltanissetta

Lecce

Vibo Valentia

Alto Belice Corleonese

Nápoli Nordeste

Barese Ofantino Norte

Sangro Aventino

Área Sur de Basilicata

Canavese

Cosentino

Ferrara

Foggia

Locride

Maremma Grossetana

Rovigo

Simeto Etna

Teramo

Valdichiana - Amiata - Trasimeno - Orvietano

Fuente: Ministero dell'Economia, Dipartimento Politiche di Sviluppo e di Coesione (2003). 